\title{
Thermal Aspects of Melanistic and Striped Morphs of the Snake Elaphe quadrivirgata
}

\author{
Koji Tanaka* \\ Department of Zoology, Graduate School of Science, Kyoto University, \\ Sakyo, Kyoto 606-8502, Japan
}

\begin{abstract}
Temperature is a critical factor limiting various aspects of the biology of ectotherms. In addition to environmental factors, coloration and body size are two physical properties that influence ectotherms' body temperature $\left(T_{\mathrm{b}}\right)$. I compared the influences of these properties on thermal aspects of the two morphs of the color-dimorphic snake (E. quadrivirgata) under experimental conditions. First, I fitted $T_{\mathrm{b}}$ data during heating to the von Bertalanffy equation, but considered parameter values of the equilibrium temperature obtained to be biologically meaningless. Alternatively, I limited the data for comparison of the morphs to $T_{\mathrm{b}} \leq 35^{\circ} \mathrm{C}$, which was the $T_{\mathrm{b}}$ at which snakes began to move vigorously in the experiment. The rate of $T_{\mathrm{b}}$ increase was significantly greater in the melanistic morph than in the striped morph. Heating rate was negatively correlated with body size in both morphs. The interaction of body size and heating rate did not significantly differ between the two morphs. The possibility of linkage, due to thermal advantage, between small body size and the prevalence of melanism in the population studied is briefly discussed. Rapid increase of $T_{\mathrm{b}}$ is biologically advantageous because snakes with such ability would be released from various time and environmental constraints associated with thermoregulation under particular environmental conditions.
\end{abstract}

Key words: body temperature, body size, coloration, Elaphe quadrivirgata, Yakushima Island

\section{INTRODUCTION}

Ectotherms rely on external resources for heat gain, and thus temperature is a critical factor limiting their distribution, diversity, and activity (e.g., Cowles and Bogert, 1944; Huey, 1982; Coxwell and Bock, 1995). Although the range of body temperature $\left(T_{\mathrm{b}}\right)$ available to them is constrained by environmental factors, they can adjust $T_{\mathrm{b}}$ by physical, physiological, and behavioral means (see Lillywhite [2001] for review). For many ectotherms, thermoregulation is of central importance in their daily life because $T_{b}$ directly affects their physiological processes, performance, and behavior (e.g., Dawson, 1975; Christian and Tracy, 1981; Hertz et al., 1983; Stevenson et al., 1985; Bennett, 1987; van Berkum, 1988; Huey and Kingsolver, 1989; Willmer, 1991; Bauwens et al., 1995; Dorcas et al., 1997; Forsman, 1999). Thus, selection should act to favor characteristics that enhance the thermoregulatory ability of ectotherms, because the latter is biologically important to them.

Coloration and body size are two physical properties that influence the $T_{\mathrm{b}}$ of ectotherms (e.g., Watt, 1968; Gibson

\footnotetext{
* Corresponding author. Phone: +81-75-753-4099;

Fax : +81-75-753-4113;

E-mail: koji@ethol.zool.kyoto-u.ac.jp
}

and Falls, 1979; Brakefield and Willmer, 1985; Stevenson, 1985; Stewart and Dixon, 1989; Forsman, 1995a, 1997; de Jong et al., 1996; Bittner et al., 2002; Forsman et al., 2002; Gross et al., 2004). These two properties are consequences of adaptive compromise among various conflicting demands, such as social, predatory, antipredatory, and thermoregulatory demands (Cooper and Greenberg, 1992). Thus, if a particular combination of coloration and size is advantageous to the thermoregulation of a certain species, and innocuous for other demands, it is possible that these two properties will be highly correlated with each other.

Snakes are a suitable experimental ectothermic animal for examining the influence of coloration and body size on $T_{\mathrm{b}}$, because they exhibit a wide range of variation in these two physical properties, yet have a simple body form (Greene, 1997). Furthermore, the occurrence of intraspecific variation in both properties in some snakes (e.g., Mitchell, 1977; Rossman et al., 1996; Tanaka and Ota, 2002) enables us to examine the relationship between coloration and body size and their effects on thermal properties without the confounding factor of phylogenetic constraints.

Among many color variants of snakes, the melanistic morph has been attractive for numerous studies that attempted to elucidate the biological significance of color mor- 
phs. Because the melanistic morph has been thought to have thermoregulatory advantages over normal-colored morphs (Gibson and Falls, 1979), studies of melanism in snakes have heavily focused on the thermal aspects. For example, Bittner et al. (2002) investigated the effects of color and body size on $T_{\mathrm{b}}$ of the garter snake, Thamnophis sirtalis, and found that the melanistic morph has a higher equilibrium temperature than the striped morph only in large size classes, whereas heating rate does not differ between the two color morphs in any size class. Based on their results and those of Shine and Kearney (2001), who investigated the effects of several attributes of a physical model (e.g., color, size) on its temperature, Bittner et al. (2002) suggested that the thermal advantages of melanism would be restricted to larger individuals. Forsman (1995a) found that when exposed to natural insolation, the melanistic morph of the adder (Vipera berus) heated faster and reached slightly higher $T_{\mathrm{b}}$ than the normal-colored morph, whereas no consistent difference occurred in daily $T_{\mathrm{b}}$ variation between free-ranging melanistic and normal-colored individuals, monitored by radiotelemetry. He concluded that either color exerted only a trivial effect on $T_{\mathrm{b}}$ of the adders, or melanistic individuals used their thermoregulatory advantage differently, depending on gender and reproductive condition (Forsman, 1995a).

Snakes are a highly diversified group of reptiles (Greene, 1997), and thus they offer the valuable advantage of comparative study of biologically dissimilar species. The Japanese four-lined snake (Elaphe quadrivirgata) is a suitable candidate in this respect because not only does it exhibit color polymorphism, including melanism (Stejneger, 1907; Goris and Maeda, 2004; Mori et al., 2005), but it also differs from the garter snake and the adder in several lifehistory traits. For example, $T$. sirtalis and $V$. berus are viviparous, whereas $E$. quadrivirgata is oviparous. In addition, the female is the larger sex in $T$. sirtalis and $V$. berus, whereas the male is the larger sex in E. quadrivirgata.

Temperatures during the developmental period affect the post-hatching behavior and morphometrics of hatchlings in several snakes (e.g., Vinegar, 1974; Osgood, 1978; Burger, 1989, 1990; Blouin-Demers et al., 2000; Lourdais et al., 2004). Precise thermoregulation is particularly critical for viviparous snakes because they retain their embryos for relatively long periods. In contrast, appropriate nest-site selection may be more critical than precise thermoregulation for oviparous snakes to produce healthy hatchlings (Plummer and Snell, 1988; Shine et al., 1997). If this dichotomy applies to $T$. sirtalis and $V$. berus compared to $E$. quadrivirgata, thermoregulatory ability is most important for the adult female garter snakes and adders (i.e., the larger sex of viviparous snakes). These biological differences may affect the relative importance of body size and coloration to the thermal aspects of each species in different ways.

Here, I investigated the effects of color and body size on thermal aspects of $E$. quadrivirgata under experimental conditions. Specifically, I compared the relationship of body size to heating rate between melanistic and normal-colored (striped) morphs.

\section{MATERIALS AND METHODS}

\section{Subject animals}

Elaphe quadrivirgata is a diurnal snake widely distributed in Japan (Stejneger, 1907; Goris and Maeda, 2004; Mori et al., 2005). The normal coloration of adult snakes is a brown ground color with four black longitudinal stripes. Color patterning is, however, variable both among and within populations (e.g., variants include yellowish ground color with vivid stripes, or dark-brown ground color with pale stripes). Melanistic snakes are black from hatching, and thus melanism is not an ontogenetic darkening of the ground color, as is seen in the adders (Naulleau, 1973; Forsman, 1995a, b).

For the past seven years, I have been conducting an ecological study of the snakes on Yakushima Island $\left(30^{\circ} 20^{\prime} \mathrm{N}, 130^{\circ} 32^{\prime} \mathrm{E}\right)$, Kagoshima Prefecture, Japan. Yakushima, a large island $\left(503 \mathrm{~km}^{2}\right.$ in area) located southwest of the main-islands of Japan, has a warm-temperate climate (annual mean air temperature approximately $20^{\circ} \mathrm{C}$ in the lowlands) with extensive precipitation ( $>2500$ $\mathrm{mm} /$ year) (Eguchi, 1985).

On Yakushima Island, E. quadrivirgata exhibits features of both coloration and body size that differ from those of main-island populations. There is a distinct striped/melanistic color dimorphism, with a high proportion of melanistic individuals (approximately $85 \%$; Tanaka and Ota, 2002). Body size is smaller than that of the mainisland populations (Fukada, 1992; Kadowaki, 1996; Tanaka and Ota, 2002). For example, mean snout-vent length (SVL) and body mass (BM) of adults on Yakushima Island are approximately $80 \%$ and $30 \%$, respectively, of those in the Kyoto population studied by Fukada (1992) (mean male SVL=1078 mm and $828 \mathrm{~mm}$ for Kyoto and Yakushima, respectively, and mean $B M=300 \mathrm{~g}$ and $114 \mathrm{~g}$, respectively; see also Tanaka and Ota [2002]).

Experimental subjects were wild-caught adults comprising 13 melanistic ( 8 males and 5 females) and 14 striped snakes ( 8 males and 6 females). All melanistic snakes and two striped females were collected from Yakushima Island. Due to the difficulty of obtaining striped individuals from Yakushima (I captured only 33 striped individuals during a 7-year ecological survey), the remaining striped snakes were collected from Shiga Prefecture $\left(34^{\circ} 55^{\prime} \mathrm{N}, 136^{\circ} 05^{\prime} \mathrm{E}\right)$, Honshu Island (the main-island of Japan). All striped snakes used in the experiment had a brown ground color with vivid stripes, and all melanistic snakes were jet-black. Although striped snakes came from two populations, the small sample size precluded the analysis of population effects on thermal properties. However, at least the appearance of the snakes did not differ between the two populations, and thus I assumed that population effects, if any, were negligible.

Prior to experimental trials, each snake was housed individually in a plastic cage $(35 \times 20 \times 15 \mathrm{~cm})$ with a water dish, in a building in which air temperature fluctuated with changes in ambient temperature throughout the experimental period (range $22-34^{\circ} \mathrm{C}$ ).

\section{Heating experiment}

As has been done in other studies (e.g., Forsman, 1997; Bittner et al., 2002), I used an artificial heat source, in this case a 100watt light bulb (Vivarium Basking Spot, Pogona Club Inc., Japan) hung $40 \mathrm{~cm}$ above the snake. This was done because experiments under natural conditions introduce many uncontrollable factors. To simulate the emergence of a snake from an overnight refugium, the heating experiment was performed in a walk-in environmental chamber at a temperature of $25^{\circ} \mathrm{C}$, which is the approximate air temperature measured when $E$. quadrivirgata was captured between 0700 and $1000 \mathrm{hr}$ on Yakushima Island $(n=82$, mean= $25.7^{\circ} \mathrm{C}, \mathrm{SE}=0.30^{\circ} \mathrm{C}$; Tanaka, unpubl. data). Snakes were not fed for 
at least 3 days prior to trials so that they could expel their gut contents.

On the day before an experimental trial, a snake was removed from its cage, put into a 5-mm mesh nylon bag, and kept in an incubator at $18^{\circ} \mathrm{C}$ until the trial began. Immediately before the trial, the snake was removed from the incubator. The small sensor bulb of a thermistor was inserted into the cloaca of the snake, and the wire of the thermistor probe was taped to its tail to prevent the sensor bulb from pulling out (Lutterschmidt and Lutterschmidt, 2002). The snake was then put into another 5-mm mesh nylon bag, which was sewn onto a $25 \times 20 \times 2.2-\mathrm{cm}$ board of styrofoam to restrict the snake's movements and to minimize inter-trial differences in position of the snake relative to the heat source. Because these handling procedures affected $T_{\mathrm{b}}$, I allowed the snake to re-equilibrate in the incubator to a $T_{b}$ of approximately $20^{\circ} \mathrm{C}$. The board with the snake was then transferred to the walk-in environmental chamber and placed under the heat source, within $30 \mathrm{sec}$ after removal from the incubator.

I turned on the heating bulb when the $T_{\mathrm{b}}$ of the snake reached approximately $21.0^{\circ} \mathrm{C}$ (time zero of a trial), and began to record $T_{\mathrm{b}}$ at 1-min intervals. The mesh of the bag was large enough that most of the radiation reached to the snake. I simultaneously recorded the behavior of the snake. I terminated each trial either after $40 \mathrm{~min}$ had elapsed or when $T_{\mathrm{b}}$ reached $40^{\circ} \mathrm{C}$, whichever came first. After the trial, I verified that the bulb sensor of the thermistor had remained in the cloaca, and I measured SVL and BM of the snake. Mean SVLs of the melanistic and striped morphs were $889 \mathrm{~mm}$ (range 780-1068 mm) and $869 \mathrm{~mm}$ (range 740-1010 mm), respectively, and mean BMs were $150 \mathrm{~g}$ (range 102-208 g) and $147 \mathrm{~g}$ (range 91-208 g), respectively. ANCOVA (with morph as factor, BM as dependent variable, and $S V L$ as covariate) revealed that neither SVL-adjusted BM (t-test, $d f=1,24, F=1.03, p=0.21$ ) nor $S V L$ (t-test, $\mathrm{df}=25, \mathrm{t}=0.53, \mathrm{p}>0.6$ ) differed between the two morphs. One to four snakes were tested in a day. After the experiment, all snakes were released at the site of capture.

\section{Data analyses}

Immediately after each day's experiments, $T_{\mathrm{b}}$ data for each snake were fitted to the following von Bertalanffy equation:

$$
T_{\mathrm{b}}=A\{1-B \exp [-C \text { (time) }]\},
$$

where $A$ is equilibrium temperature, $B$ is proportion of $A$ realized at time $=0$, and $C$ is a heating coefficient, which has units of time ${ }^{-1}$. If data from a snake failed to converge on this equation due to a constant rise in $T_{\mathrm{b}}$ throughout a trial, the snake was re-tested another day.

Homogeneity of variance was checked with Bartlett's test before parametric tests were adopted. All statistical analyses were conducted with JMP (version 3) statistical software (SAS Institute, Inc., 1995) with $\alpha=0.05$. Data are presented as mean $\pm 1 S E$.

\section{RESULTS}

Equilibrium temperatures obtained by the von Bertalanffy equation exceeded $50^{\circ} \mathrm{C}$ (Table 1). On Yakushima, operative environmental temperatures (Bakken and Gates, 1975;
Bakken, 1992) under full sun during the active seasons of the snake frequently exceed $50^{\circ} \mathrm{C}$ (Tanaka, unpubl. data), and thus the heating curve may well represent changes in $T_{\mathrm{b}}$ of a basking snake in full sun. However, $T_{\mathrm{b}}$ of free-ranging snakes on Yakushima never exceeds $35^{\circ} \mathrm{C}$ (Tanaka, unpubl. data), and the equilibrium temperatures obtained were obviously much higher than a lethal temperature. Thus, detailed analyses of equilibrium temperatures exceeding $50^{\circ} \mathrm{C}$ are biologically meaningless. Nonetheless, this does not necessary mean that the heating curve obtained is irrelevant. Topology of the heating curve may differ between free-ranging and experimental conditions only above a particular $T_{\mathrm{b}}$ at which a basking snake in the wild would begin to move to another place. I assumed this point to be $35^{\circ} \mathrm{C}$, for the following reasons. First, in the experiment, most snakes of both morphs began to move vigorously, with tongue flicking, when their $T_{\mathrm{b}}$ reached approximately $35^{\circ} \mathrm{C}$. Second, the maximum $T_{\mathrm{b}}$ of $E$. quadrivirgata measured in the field on Yakushima Island was $34.6^{\circ} \mathrm{C}$ (Tanaka, unpubl. data). Third, the maximum voluntary $T_{\mathrm{b}}$ of $E$. quadrivirgata recorded under a laboratory thermal gradient was $34.9^{\circ} \mathrm{C}$ (Tanaka, unpubl. data). Thus, in the following analyses, I limited $T_{\mathrm{b}}$ data to no higher than $35^{\circ} \mathrm{C}$ in order to examine heating during basking (i.e., from the beginning of basking until moving to another place). Use of linear regression equation was justified in because the exponential curves represented by the von Bertalanffy equation approximate a straight line (all, $r>0.96, p<0.001$; Fig. 1) during the presumed basking stage (i.e., $T_{b} \leq 35^{\circ} \mathrm{C}$ ).

Heating rate (slope of $T_{\mathrm{b}}$ against time) during the presumed basking stage did not significantly differ between males and females in both morphs (melanistics: $d f=11$, $\mathrm{t}=1.00, \mathrm{p}=0.34$; striped: $d f=12, \mathrm{t}=1.38, \mathrm{p}=0.19$ ). Thus, I pooled the data from both sexes in the following analyses. Heating rate was negatively correlated with SVL (melanistics: $r=-0.592, p=0.03$; striped: $r=-0.58, p=0.03$ ) and BM (melanistics: $r=-0.65, p=0.02$; striped: $r=-0.55, p=0.04$; Fig. 2 ) in both morphs. ANCOVA (with morph as factor, BM as covariate, and heating rate as dependent variable) showed a significant difference in intercept between the two morphs, but no significant difference in slope (intercept: $\mathrm{df}=1,24, \mathrm{~F}=7.43$, $\mathrm{p}=0.01$; slope: $\mathrm{df}=1,23, \mathrm{~F}=2.24, \mathrm{p}=0.15$ ), although the slope was steeper in the melanistic morph $(-0.00272)$ than in the striped morph $(-0.00113)$ (use of SVL as covariate yielded the same conclusions). This means that the melanistic morph heats faster than the striped morph, but that the effect of BM (or SVL) on heating rate does not differ between the two morphs. These results did not change even when I used

Table 1. Mean $\pm 1 S E$ of parameter values of the von Bertalanffy equation fitted to body temperature data during heating for melanistic and striped morphs of Elaphe quadrivirgata. Ranges are given in parentheses. $\mathrm{N}=$ sample sizes.

\begin{tabular}{llll}
\hline Morph & $\mathrm{N}$ & Equilibrium temperature $\left({ }^{\circ} \mathrm{C}\right)$ & Heating coefficient $\left(\mathrm{min}^{-1}\right)$ \\
\hline Melanistic & 13 & $50.3 \pm 3.4(40.3-86.5)$ & $0.0304 \pm 0.0038(0.0085-0.0656)$ \\
Striped & 14 & $53.4 \pm 3.3(38.9-79.8)$ & $0.0238 \pm 0.0035(0.0071-0.0470)$ \\
\hline
\end{tabular}




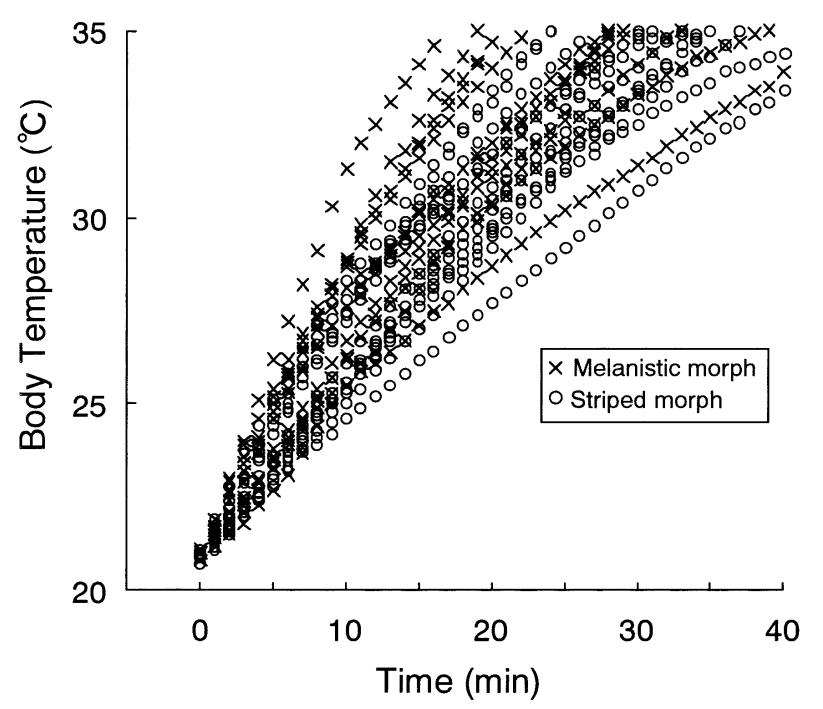

Fig. 1. Plots of body temperature $\left(T_{\mathrm{b}}\right)$ against time during the presumed basking stage (from the beginning of a trial until $T_{\mathrm{b}}$ reached $35^{\circ} \mathrm{C}$ ) for 27 individuals of Elaphe quadrivirgata (13 melanistic and 14 striped morphs). The regression line for each individual is not shown (all, $r>0.96, p<0.001$ ).

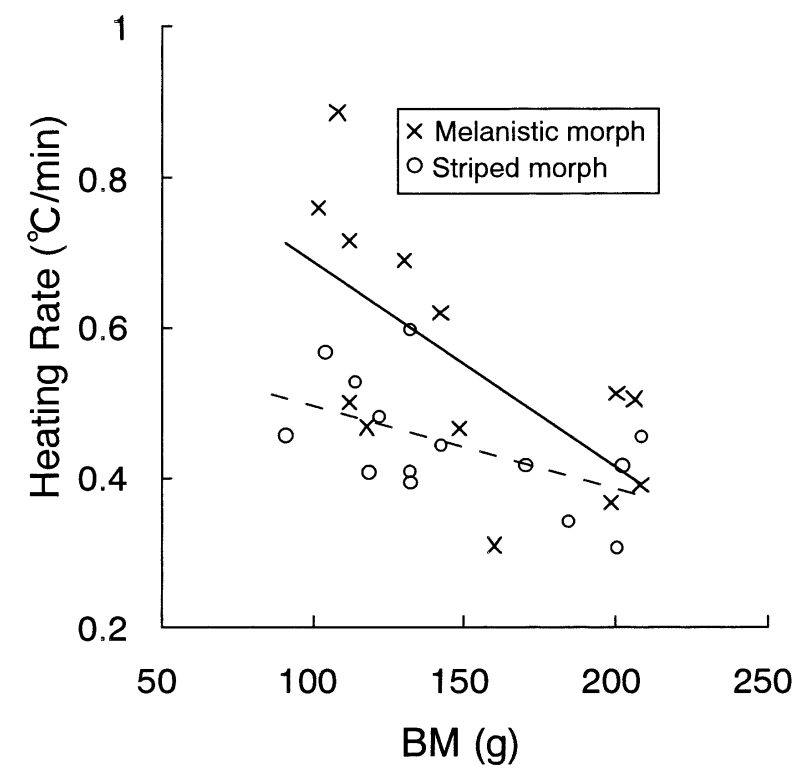

Fig. 2. Relationships between body mass (BM) and heating rate during the presumed basking stage (from the beginning of a trial until $T_{\mathrm{b}}$ reached $35^{\circ} \mathrm{C}$ ) for melanistic and striped morphs of Elaphe quadrivirgata. Solid and dashed lines show least-squares regression lines fitted to the melanistic and striped morphs, respectively. Regression equation for melanistic morph: Heating rate $=0.95962-$ $0.00272 \mathrm{BM}\left(r^{2}=0.425\right)$. For striped morph: Heating rate $=0.60976$ $0.00113 \mathrm{BM}\left(r^{2}=0.300\right)$.

another $T_{\mathrm{b}}$ value, such as the upper set-point (75\% quartile; Hertz et al., 1993) or median selected $T_{\mathrm{b}}$ under the thermal gradient, as the end point $\left(31.6^{\circ} \mathrm{C}\right.$ and $30.2^{\circ} \mathrm{C}$, respectively; Tanaka, unpubl. data). The melanistic morph attained a $T_{\mathrm{b}}$ of $35^{\circ} \mathrm{C}$ significantly faster than the striped morph (melanistic: 27.0 $\pm 2.1 \mathrm{~min}$, range 16-40 min; striped: $32.4 \pm 1.4 \mathrm{~min}$, range
24-40 $\mathrm{min} ; \mathrm{df}=25, \mathrm{t}=-2.18, \mathrm{p}=0.04)$.

\section{DISCUSSION}

The heating rate during the presumed basking stage was negatively correlated with body size in both morphs. This result corresponds with those of previous empirical and theoretical studies (e.g., McNab and Auffenberg, 1976; Stevenson, 1985; Turner and Tracy, 1985; Seebacher et al., 1999). An interesting trend in relation to heating rate and body size shown by the present study is that the slope of the regression equation for heating rate against $\mathrm{BM}$ is steeper in the melanistic morph than in the striped morph, although the difference is not statistically significant. This statistical non-significance may be due to the narrow range of body size used in the experiment or to small sample sizes. A larger inter-morph difference in heating rate in small snakes than in large snakes may be key to explaining the small body size and high frequency of melanism on Yakushima Island. Considering the superior heat absorption by black color, it may be possible that small body size helps maintain this prevalence of melanism.

A high proportion of melanistic morphs in dwarf populations of E. quadrivirgata also occurs on Oh-shima Island, located off the south coast of central Honshu Island (Hasegawa and Moriguchi, 1989; Goris and Maeda, 2004). The body size of snakes is geographically highly plastic, and has primarily been considered to be a direct phenotypic response to local prey type and size (Schwaner, 1985; Hasegawa and Moriguchi, 1989; Forsman, 1991; Kohno and Ota, 1991; Mori, 1994; Tanaka and Ota, 2002). Thus, the body size trend and the origin and maintenance of color dimorphism within a population must be maintained by independent mechanisms, but a secondary linkage between small size and a high frequency of melanism may exist on the basis of thermal advantage. Similarly to $E$. quadrivirgata in the present study, melanism usually prevails in species of ladybird beetles small in body size (Stewart and Dixon, 1989), although interpretation of this observation contrasts with that presented here (i.e., advantage of rapid heating in small snakes vs. disadvantage of overheating in large ladybird beetles). From the perspective of evolutionary biology, direct and indirect links between morphology, physiology, behavior, and fitness are a fruitful area for future study (Willmer, 1991; Garland and Losos, 1992).

Under natural insolation, the melanistic morph of the garter snake maintains higher $T_{\mathrm{b}}$ than the striped morph (Gibson and Falls, 1979), and the melanistic morph of the adder heats faster and reaches slightly higher $T_{\mathrm{b}}$ than the normal-colored morph (Forsman, 1995a). The melanistic morph of $E$. quadrivirgata also heats faster than the striped morph. Rapid attainment of the preferred range of $T_{b}$ is advantageous for ectotherms. This ability releases snakes from various time and environmental constraints associated with thermoregulation. For example, rapid heating enables a snake to utilize intermittently available, short-duration sun as 
a heat resource. Additionally, if thermally suitable sunlit sites are rare and appear patchily, and thus active movement is required for every basking effort, slow heaters must waste more time in thermoregulation than rapid heaters. This is because slow heaters may be unable to reach a $T_{\mathrm{b}}$ within their preferred range during a patch of clear sky. Furthermore, slow heaters may be restricted in their activities to the vicinity of thermally suitable microhabitats. The snake population on which I focused inhabits secondary forests, and the snakes may not be able to attain their preferred $T_{\mathrm{b}}$ by random use of microhabitats, except during summer (Tanaka, in prep.). In addition, unsettled weather conditions and high rainfall are well known climatic features of Yakushima Island (Eguchi, 1985). Thus, the hypothetical situations presented above are feasible ones.

In the wild, there are four possible ways in which slow heaters could manage their thermal inferiority. First, they could maintain a level of thermoregulation similar to that of melanistic morphs and accept a suboptimal $T_{\mathrm{b}}$ for activities. Second, they could turn into thermoconformers. Third, they could devote the time required and restrict other activities to the vicinity of a specific habitat to attain a $T_{\mathrm{b}}$ comparable to fast heaters. Fourth, they could lower their preferred $T_{\mathrm{b}}$. To verify the ecological relevance of a rapid increase in $T_{b}$ and its consequences, field studies of the thermal biology of $E$. quadrivirgata on Yakushima Island using temperature-sensitive radio transmitters will be necessary.

While some studies were consistent with the present study (see above), Bittner et al. (2002) reported inconsistent results. They revealed that when exposed to a light-bulb heat source, large melanistic garter snakes exhibited a higher equilibrium temperature than large striped ones, but heating rate did not differ between the two morphs. Interspecific differences in some characteristics (e.g., physiological differences, differences in skin properties; also see Introduction) are possible causes of the inconsistency, however, a detailed discussion is impossible due to the lack of direct comparative data.

Lastly, I should mention the remarkable variance of $T_{\mathrm{b}}$ among individuals at a given time (see Fig. 1). It is obviously due, in part, to variation in body size (BM explained $42.5 \%$ and $30.0 \%$ of the total variation in heating rate for melanistic and striped morphs, respectively). In addition, either undetermined factor(s) or subtle differences in behavioral and physiological responses (see Bartholomew [1982]; Lillywhite [2001] for reviews) among individual snakes might have influenced their $T_{\mathrm{b}}$ change in complicated ways.

The present study revealed that physical properties play an important role in the heating of $E$. quadrivirgata. To validate the thermal superiority of the melanistic morph in an ecological context, it would be useful to study the differences between the two morphs in when, where, and how they behaviorally regulate their $T_{b}$ in response to thermal environments.

\section{ACKNOWLEDGMENTS}

I am grateful to A. Mori for his valuable comments and suggestions on the manuscript and to $\mathrm{H}$. Ota for first introducing of me to the topic of color dimorphism in E. quadrivirgata on Yakushima Island. I thank many inhabitants of Yakushima Island and researchers for their hospitality during my stays at the Kyoto University Yakushima Field Station. Data analyses were partially supported by a Grant for Biodiversity Research of the 21st Century COE (A14).

\section{REFERENCES}

Bakken GS (1992) Measurement and application of operative and standard operative temperatures in ecology. Am Zool 32: 194216

Bakken GS, Gates DM (1975) Heat-transfer analysis of animals: Some implications for field ecology, physiology, and evolution. In "Perspectives of Biophysical Ecology" Ed by DM Gates, RB Schmerl, Springer-Verlag, New York, pp 255-290

Bartholomew GA (1982) Physiological control of body temperature. In "Biology of the Reptilia Vol 12" Ed by C Gan, FH Pough, Academic Press, New York, pp 167-211

Bauwens D, Garland T Jr, Castilla AM, Van Damme R (1995) Evolution of sprint speed in lacertid lizards: Morphological, physiological, and behavioral covariation. Evolution 49: 848-863

Bennett AF (1987) Evolution of the control of body temperature: Is warmer better? In "Comparative Physiology: Life in Water and on Land" Ed by P Dejours, L Bolis, CR Taylor, ER Weibel, Liviana Press, Padova, pp 421-431

Bittner TD, King RB, Kerfin JM (2002) Effects of body size and melanism on the thermal biology of garter snakes (Thamnophis sirtalis). Copeia 2002: 477-482

Blouin-Demers G, Kissner KJ, Weatherhead PJ (2000) Plasticity in preferred body temperature of young snakes in response to temperature during development. Copeia 2000: 841-845

Brakefield PM, Willmer PG (1985) The basis of thermal melanism in the ladybird Adalia bipunctata: Differences in reflectance and thermal properties between the morphs. Heredity 54: 9-14

Burger J (1989) Incubation temperature has long-term effects on behaviour of young pine snakes (Pituophis melanoleucus). Behav Ecol Sociobiol 24: 201-207

Burger J (1990) Effects of incubation temperature on behavior of young black racers (Coluber constrictor) and kingsnakes (Lampropeltis getulus). J Herpetol 24: 158-163

Christian KA, Tracy CR (1981) The effect of the thermal environment on the ability of hatchling Galapagos land iguanas to avoid predation during dispersal. Oecologia 49: 218-223

Cooper WE Jr, Greenberg N (1992) Reptilian coloration and behavior. In "Biology of the Reptilia Vol 18" Ed by C Gans, D Crews, University of Chicago Press, Chicago, pp 298-422

Cowles RB, Bogert CM (1944) A preliminary study of the thermal requirements of desert reptiles. Bull Am Mus Nat Hist 83: 261296

Coxwell CC, Bock CE (1995) Spatial variation in diurnal surface temperatures and the distribution and abundance of an alpine grasshopper. Oecologia 104: 433-439

Dawson WR (1975) On the physiological significance of the preferred body temperatures of reptiles. In "Perspectives of Biophysical Ecology" Ed by DM Gates, RB Schmerl, SpringerVerlag, New York, pp 443-473

de Jong PW, Gussekloo SWS, Brakefield PM (1996) Differences in thermal balance, body temperature and activity between non-melanic and melanic two-spot ladybird beetles (Adalia bipunctata) under controlled conditions. J Exp Biol 199: 26552666 
Dorcas ME, Peterson CR, Flint MET (1997) The thermal biology of digestion in rubber boas (Charina bottae): Physiology, behavior, and environmental constraints. Physiol Zool 70: 292-300

Eguchi T (1985) Climate of Yaku-shima Island, especially regionality of precipitation distribution. In "Conservation Reports of the Yakushima Wilderness Area, Kyushu, Japan. May 1984" Nature Conservation Bureau, Environment Agency, Tokyo, pp 3-26 (in Japanese, with English abstract)

Forsman A (1991) Variation in sexual size dimorphism and maximum body size among adder populations: Effects of prey size. J Anim Ecol 60: 253-267

Forsman A (1995a) Heating rates and body temperature variation in melanistic and zigzag Vipera berus: Does colour make a difference? Ann Zool Fenn 32: 365-374

Forsman A (1995b) Opposing fitness consequences of colour pattern in male and female snakes. J Evol Biol 8: 53-70

Forsman A (1997) Thermal capacity of different colour morphs in the pygmy grasshopper Tetrix subulata. Ann Zool Fenn 34: 145-149

Forsman A (1999) Variation in thermal sensitivity of performance among colour morphs of a pygmy grasshopper. J Evol Biol 12: 869-878

Forsman A, Ringblom K, Civantos E, Ahnesjö J (2002) Coevolution of color pattern and thermoregulatory behavior in polymorphic pygmy grasshoppers Tetrix undulata. Evolution 56: 349-360

Fukada H (1992) Snake Life History in Kyoto. Impact Shuppankai, Tokyo

Garland T Jr, Losos JB (1992) Ecological morphology of locomotor performance in squamate reptiles. In "Ecological Morphology: Integrative Organismal Biology" Ed by PC Wainwright, SM Reilly, Chicago University Press, Chicago, pp 240-302

Gibson AR, Falls JB (1979) Thermal biology of the common garter snake Thamnophis sirtalis (L.) II. The effects of melanism. Oecologia 43: 99-109

Goris RC, Maeda N (2004) Guide to the Amphibians and Reptiles of Japan. Krieger Publishing Company, Malabar

Greene HW (1997) Snake: The Evolution of Mystery in Nature. University of California Press, California

Gross J, Schmolz E, Hilker M (2004) Thermal adaptations of the leaf beetle Chrysomela lapponica (Coleoptera: Chrysomelidae) to different climes of Central and Northern Europe. Environ Entomol 33: 799-806

Hasegawa M, Moriguchi H (1989) Geographic variation in food habits, body size and life history traits of the snakes on the Izu Islands. In "Current Herpetology in East Asia" Ed by M Matsui, T Hikida, RC Goris, Herpetol Soc Japan, Kyoto, pp 414-432

Hertz PE, Huey RB, Nevo E (1983) Homage to Santa Anita: Thermal sensitivity of sprint speed in agamid lizards. Evolution 37: 1075-1084

Hertz PE, Huey RB, Stevenson RD (1993) Evaluating temperature regulation by field-active ectotherms: The fallacy of the inappropriate question. Am Nat 142: 796-818

Huey RB (1982) Temperature, physiology and the ecology of reptiles. In "Biology of the Reptilia Vol 12" Ed by C Gans, FH Pough, Academic Press, New York, pp 25-91

Huey RB, Kingsolver JG (1989) Evolution of thermal sensitivity of ectotherm performance. TREE 4: 131-135

Kadowaki S (1996) Ecology of a Japanese snake community: Resource use patterns of the three sympatric snakes, Rhabdophis tigrinus, Elaphe quadrivirgata and Agkistrodon b. blomhoffii. Bull Tsukuba Univ Forests 12: 77-148 (in Japanese, with English abstract)

Kohno H, Ota H (1991) Reptiles in a seabird colony: Herpetofauna of Nakanokamishima Island of the Yaeyama group, Ryukyu archipelago. Isl Stud Okinawa 9: 73-89

Lillywhite HB (2001) Temperature, energetics, and physiological ecology. In "Snakes: Ecology and Evolutionary Biology" Ed by RA Seigel, JT Collins, SS Novak, Blackburn Press, New Jersey, pp 422-477

Lourdais O, Shine R, Bonnet X, Guillon M, Naulleau G (2004) Climate affects embryonic development in a viviparous snake, Vipera aspis. Oikos 104: 551-560

Lutterschmidt DI, Lutterschmidt WI (2002) Modifications for the successful use of thermocouples in studies of thermoregulation. Herpetol Rev 33: 110-112

McNab BK, Auffenberg W (1976) The effect of large body size on the temperature regulation of the Komodo dragon, Varanus komodoensis. Comp Biochem Physiol 55A: 345-350

Mitchell JC (1977) Geographic variation of Elaphe guttata (Reptilia: Serpentes) in the Atlantic Coastal Plain. Copeia 1977: 33-41

Mori A (1994) Ecological and morphological characteristics of the Japanese rat snake, Elaphe climacophora, on Kammuri-jima Island: A possible case of insular gigantism. Snake 26:11-18

Mori A, Tanaka K, Moriguchi H, Hasegawa M (2005) Color variations in Elaphe quadrivirgata throughout Japan. Bull Herpetol Soc Jpn 2005: 22-38 (in Japanese)

Naulleau G (1973) Le mélanisme chez Vipera aspis et chez Vipera berus. Bull Soc Zool France 98: 595-596 (in French)

Osgood DW (1978) Effects of temperature on the development of meristic characters in Natrix fasciata. Copeia 1978: 33-47

Plummer MV, Snell HL (1988) Nest site selection and water relations of eggs in the snake, Opheodrys aestivus. Copeia 1988: 58-64

Rossman DA, Ford NB, Seigel RA (1996) The Garter Snakes: Evolution and Ecology. University of Oklahoma Press, Norman

SAS Institute Inc (1995) JMP Statistics and Graphics Guide. SAS Institute Inc, Cary, North Carolina

Schwaner TD (1985) Population structure of black tiger snakes, Notechis ater niger, on offshore islands of South Australia. In "The Biology of Australasian Frogs and Reptiles" Ed by G Grigg, R Shine, H Ehmann, Royal Zoological Society of New South Wales, Sydney, pp 35-46

Seebacher F, Grigg GC, Beard LA (1999) Crocodiles as dinosaurs: Behavioural thermoregulation in very large ectotherms leads to high and stable body temperatures. J Exp Biol 202: 77-86

Shine R, Kearney M (2001) Field studies of reptile thermoregulation: How well do physical models predict operative temperature? Funct Ecol 15: 282-288

Shine R, Madsen TRL, Elphick MJ, Harlow PS (1997) The influence of nest temperatures and maternal brooding on hatchling phenotypes in water pythons. Ecology 78: 1713-1721

Stejneger L (1907) Herpetology of Japan and adjacent territory. Bull US Nat Mus 58: 1-577

Stevenson RD (1985) Body size and limits to the daily range of body temperature in terrestrial ectotherms. Am Nat 125: 102117

Stevenson RD, Peterson CR, Tsuji JS (1985) The thermal dependence of locomotion, tongue flicking, digestion, and oxygen consumption in the wandering garter snake. Physiol Zool 58: 46-57

Stewart LA, Dixon AFG (1989) Why big species of ladybird beetles are not melanic. Funct Ecol 3: 165-171

Tanaka K, Ota H (2002) Natural history of two colubrid snakes, Elaphe quadrivirgata and Rhabdophis tigrinus, on Yakushima Island, southwestern Japan. Amphibia-Reptilia 23: 323-331

Turner JS, Tracy CR (1985) Body size and the control of heat exchange in alligators. J Therm Biol 10: 9-11

van Berkum FH (1988) Latitudinal patterns of the thermal sensitivity of sprint speed in lizards. Am Nat 132: 327-343

Vinegar A (1974) Evolutionary implications of temperature induced anomalies of development in snake embryos. Herpetologica 30: $72-74$

Watt B (1968) Adaptive significance of pigment polymorphisms in 
Colias butterflies. I. Variation of melanin pigment in relation to thermoregulation. Evolution 22: 437-458
Willmer $P$ (1991) Thermal biology and mate acquisition in ectotherms. TREE 6: 396-399

(Received March 23, 2005 / Accepted August 1, 2005) 